\title{
Práticas de enfermagem na promoção da saúde do homem: revisão integrativa
}

\author{
Prácticas de enfermería en la promoción de la salud del hombre: una revisión \\ integradora
}

Nursing care of men's health promotion: a integrative review

Mágno Rodrigues de Carvalho ${ }^{1}$, Jéssica de Moura Caminha1, Ellen Eduarda Santos Ribeiro ${ }^{*}$, Ivanilda Sepúlveda Gomes ${ }^{1}$, Simone Santos e Silva Melo¹.

\section{RESUMO}

Objetivo: Identificar estratégias e intervenções de promoção da saúde destinadas à população masculina bem como analisar as principais temáticas abordadas. Metodologia: Trata-se de revisão integrativa no qual buscaram-se as publicações nas bases CINAHL, MEDLINE e LILACS, do período de 2010 a 2017. Nesse sentido os descritores (em português) da pesquisa são: saúde do homem, ensino, educação médica, educação em enfermagem, educação em saúde, e ocorreram por análise de resumos encontrados pela combinação dos buscadores. Ao final foram encontradas 73 publicações das quais 18 foram analisadas. Resultados: Apontaram que das 18 publicações em 9 (50,0\%) não houveram intervenção educativa e nas demais houve. As publicações foram organizadas em três eixos temáticos: Saúde do homem, Intervenções educativas direcionadas a população masculina e Ações direcionadas ao contexto profissional. Considerações finais: $O$ conhecimento dos temas mais comuns na literatura nacional e mundial relacionados a saúde é de fundamental importância para a elaboração e execução de ações de educação em saúde capazes promover uma mudança de comportamento do público masculino no tocante a sua saúde.

Palavras chave: Saúde do Homem, Educação em Saúde, Promoção da Saúde.

\begin{abstract}
Objective: Identify health promotion strategies and interventions for the male population as well as to analyze the main themes addressed. Methodology: It is an integrative review in which the publications CINAHL, MEDLINE and LILACS were searched from the period 2010 to 2017. In this sense the key terms (in Portuguese) of the research are: human health, teaching, medical education, education in nursing, health education, and occurred by analysis of summaries found by the combination of search engines. In the end, 73 publications were found, of which 18 were analyzed. Results: Pointed out that of the 18 publications in 9 $(50.0 \%)$ there was no educational intervention and in the others there was. The publications were organized in three thematic axes: Man's health, Educational interventions directed to the male population and Actions directed to the professional context. Final considerations: The knowledge of the most common themes in the national and global literature related to health is of fundamental importance for the elaboration and execution of actions of health education capable of promoting a change in the behavior of the masculine public with regard to their health
\end{abstract}

Keywords: Men's Health, Health Education, Health Promotion, Nursing Education.

${ }^{1}$ Universidade Federal do Piauí (UFPI), Teresina-Piauí. *E-mail: ellenribeirosr@gmail.com

SUBMETIDO EM: 2/2019

ACEITO EM: $3 / 2019$

PUBLICADO EM: 7/2019

REAS/EJCH | Vol.Sup.26 | e460 | DOI: https://doi.org/10.25248/reas.e460.2019 Página 1 de 9 


\section{RESUMEN}

Objetivo: Identificar estrategias e intervenciones de promoción de la salud destinadas a la población masculina, así como analizar las principales temáticas abordadas. Metodología: Se trata de una revisión integrativa en la que se buscaron las publicaciones en las bases CINAHL, MEDLINE y LILACS, del período de 2010 a 2017. En ese sentido los términos claves (en portugués) de la investigación son: salud del hombre, enseñanza, educación médica, educación en enfermería, educación en salud, y ocurrieron por análisis de resúmenes encontrados por la combinación de los buscadores. Al final se encontraron 73 publicaciones de las cuales 18 fueron analizadas. Resultados: Apuntaron que de las 18 publicaciones en $9(50,0 \%)$ no hubo intervención educativa y en las demás hubo. Las publicaciones se organizaron en tres ejes temáticos: Salud del hombre, intervenciones educativas dirigidas a la población masculina y Acciones dirigidas al contexto profesional. Consideraciones finales: El conocimiento de los temas más comunes en la literatura nacional y mundial relacionados con la salud es de fundamental importancia para la elaboración y ejecución de acciones de educación en salud capaces de promover un cambio de comportamiento del público masculino en cuanto a su salud.

Palabras clave: Salud del Hombre, Educación en Salud, Promoción de la Salud, Educación en Enfermería.

\section{INTRODUÇÃO}

Os primeiros estudos relacionados a saúde do homem datam da década de 70 , estes em sua maioria abrangiam os riscos para esta clientela de forma generalista. Com o passar dos anos os pesquisadores foram relacionando os fatores de riscos que envolvem a saúde na população masculina com enfoque de gênero (MOURA EC, et al.,2017).

O antigo modelo de atenção direcionado a quatro grupos populacionais: crianças, adolescentes, mulheres e idosos mostrou-se insuficiente para o atendimento das necessidades de saúde do Brasil, especialmente, por desconsiderar a existência de 40 milhões de homens com a faixa etária de 25 a 59 anos. Assim, a Política Nacional de Atenção Integral à Saúde do Homem (PNAISH) foi estabelecida com o objetivo de facilitar e ampliar o acesso da população masculina aos serviços de saúde e tem a educação como um de seus preceitos (BRASIL, 2009).

De acordo com Stourino LP, et al. (2013); pesquisas apontam que a expectativa de vida mundial para a população masculina é menor, média de 66 anos, quando comparado a expectativa de vida mundial feminina, média de 71 anos. Em relação à mortalidade no Brasil, dados mostram que em 2010 as chances de um homem adulto jovem morrer eram 4,5 vezes maiores quando comparado a uma mulher de mesma faixa etária e a principal causa de morte em homens foram as causas externas: acidentes, ferimento por arma de fogo e/ou branca, entre outros.

Esses dados revelam que o público masculino possui diversas particularidades que influenciam no processo saúde doença e que precisam ser compreendidas considerando sua forma de inserção na sociedade para ser possível observar os diversos perfis e padrões típicos de saúde e doença que se manifestam nesse grupo social e compreender os determinantes que operam sobre ele (STOURINO LP, et al., 2013).

Segundo Bertolini DNP e Simonetti JPO (2014); a população masculina está mais propícia a ter uma morte prematura quando comparado população feminina, devido ao modo de vida que estão inseridos. As principais causas de óbito dos homens no Brasil na faixa etária entre 20 e 59 anos foram: causas externas, doenças do aparelho circulatório, neoplasias e doenças do aparelho digestivo.

Ademais, existem questões de gênero que contribuem para este perfil de saúde, como a necessidade culturalmente enraizada de parecerem mais fortes do que as mulheres. Além desta concepção, os homens reconhecem as Unidades Básicas de Saúde (UBS) como espaços feminilizados, visto que a maioria do 
público que é atendida e os profissionais de saúde que atuam nestes locais são compostos essencialmente pelo sexo feminino. Vindicam, também, que o sistema de atenção à saúde não desenvolve ações específicas direcionadas a eles (VIEIRA K, et al., 2013).

Com isso, a Organização Mundial de Saúde (OMS) sustentou o desenvolvimento de ações voltadas para o controle e melhoria dos determinantes de saúde dos homens.

Nesse processo, a educação em saúde se revela como ferramenta chave para atuação por meio de linhas de cuidado que resguardam a integralidade das intervenções, contribuindo para o aumento da expectativa de vida e redução dos índices de adoecimento e mortes por causas evitáveis (WHO, 2009; POZZATI R, et al, 2013).

Diante do exposto, o objetivo do estudo foi identificar estratégias e intervenções de promoção da saúde destinadas à população masculina bem como analisar as principais temáticas abordadas na literatura.

\section{METODOLOGIA}

Trata-se de uma revisão integrativa para a identificação de publicações temáticas, estratégias e intervenções de promoção da Saúde do Homem no período de 2010 a 2017. Esse período se justifica por ser posterior as prerrogativas da Política de atenção à população foco no Brasil.

O estudo considerou as etapas propostas por Mendes K, et al. (2008): definição do tema e formulação do objetivo e da questão norteadora; estabelecimento dos critérios para inclusão de estudos e busca na literatura; definição das informações a serem extraídas dos estudos selecionados; avaliação dos estudos incluídos; interpretação dos resultados; apresentação da revisão/síntese do conhecimento.

A formulação da pergunta foi inspirada na estratégia PICO, definindo como a população "homens" e intervenção como "intervenções educativas na saúde do homem". O critério de comparação não foi aplicado e os resultados foram analisados sob uma perspectiva exploratória. A pergunta de pesquisa norteadora desse estudo foi: "Quais as intervenções e temáticas abordadas nas ações de promoção da saúde dos homens por meio de ações educativas?

Assim, a estratégia de identificação e seleção de estudos consistiu na busca de publicações indexadas nas bases de dados Cumulative Index to Nursing \& Allied Health Literature (CINAHL), Medical Literature and Retrivial System on Line (MEDLINE) e Literatura Latino-Americana em Ciências da Saúde (LILACS), no mês de outubro de 2017, sendo acessadas por meio do portal da Coordenação de Aperfeiçoamento de Pessoal de Nível Superior (CAPES).

Foram adotados os seguintes critérios de seleção dos artigos: estudos primários e com textos completos disponíveis; que constituíram pesquisas aplicadas em seres humanos, com abordagem de temáticas de saúde do homem e apresentaram intervenções educativas neste âmbito; publicados nacional e internacionalmente no período de 2010 a 2017, nos idiomas inglês, espanhol e português; e que atenderam à faixa etária de 25 a 59 anos.

O critério de exclusão dos artigos foi: estudos que não atendessem aos critérios de inclusão mencionados e consistissem em teses, dissertações e notícias jornalísticas.

Para a busca específica nas bases de dados, foram utilizados os descritores: Saúde do Homem, Educação em Saúde e a Promoção da Saúde, os quais apresentaram o mesmo formato para os Descritores em Ciências da Saúde (DeCS) e Medical Subject Headings (MeSH), conforme (Figura 1).

Após a aplicação dos critérios de inclusão e exclusão, foram analisados 18 artigos que embasaram a discussão. Procedeu-se a análise substancial para caracterização e extração das informações abordadas em cada artigo de interesse para o estudo.

Os trabalhos foram agrupados por similaridade de conteúdo, sob formas empíricas, sendo elaboradas três categorias para análise, assim especificadas: saúde do homem, intervenções educativas direcionadas a população masculina, e intervenções educativas no contexto profissional. 
Figura 1 - Fluxograma de seleção dos artigos encontrados. Teresina, PI, Brasil. 2017.

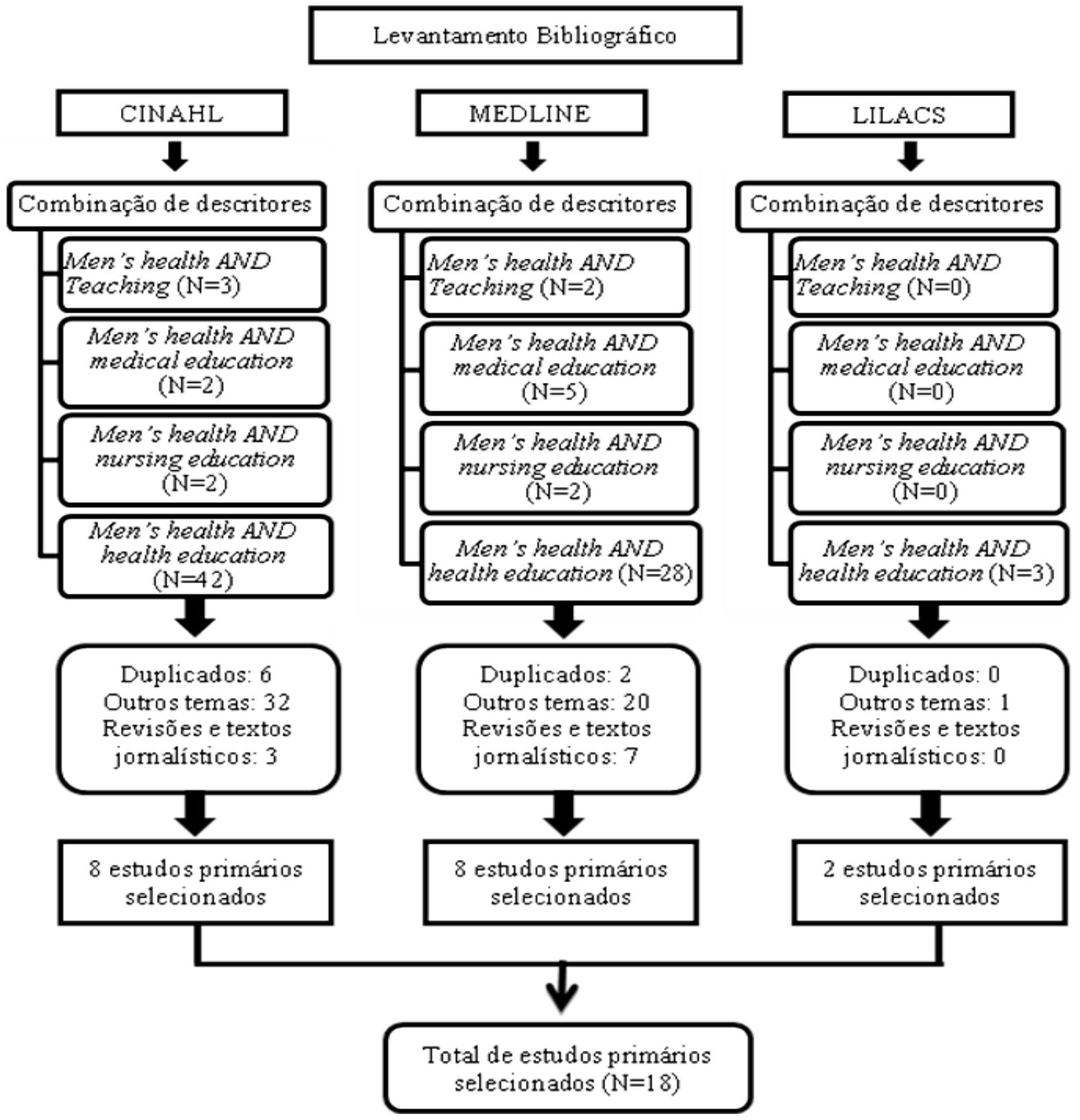

Fonte: dados da pesquisa de 2017.

\section{RESULTADOS}

Foram selecionados 18 artigos, sendo a maioria encontrada pela combinação dos termos Men's Health and Health Education 14 (77,8\%), especialmente, no portal Medline $8(44,4 \%)$ e CINAHL $8(44.4 \%)$. Foi constatado que quase a totalidade dos artigos foi publicada em periódicos estrangeiros 15 (83,3\%), distribuídos nos Estados Unidos 6 (33,3\%), Austrália 5 (27,8\%), Canadá $2(11,0 \%)$, Inglaterra $1(5,6 \%)$ e Nova Zelândia $1(5,6 \%)$, e apenas $3(16,7 \%)$ em periódicos brasileiros.

As categorias profissionais envolvidas na produção desses trabalhos foram Medicina $11(61,1 \%)$, Enfermagem $6(33,3 \%)$ e Fisioterapia 1 (5,6\%). Na classificação dos estudos, quanto ao contexto em que se realizaram, houve proporção semelhante entre artigos sem intervenção 9 (50,0\%) e com intervenção educativa 9 (50\%). Dentre estes, $7(77,8 \%)$ concentraram-se em ações de conscientização voltadas para os próprios homens e $2(22,2 \%)$ direcionaram-se a alunos e profissionais para a prestação de cuidados de qualidade na saúde do homem. 


\section{DISCUSSÃO}

\section{Saúde do homem}

Este eixo engloba 9 artigos que trataram de temas relacionados à saúde dos homens. Merecem destaque os seguintes: patologias com 5 artigos (55,5\%) e promoção da saúde com 3 artigos (33,3\%), entretanto, o aspecto masculinidade foi citado em $66,7 \%$ das publicações como influenciador das relações subjetivas que o homem estabelece com a própria saúde.

Nesta perspectiva, há um crescente reconhecimento de que a construção social da masculinidade é um fator importante que influencia na saúde tanto física quanto psíquica dos homens (MACHIN R, et al., 2011).

Segundo Yarnell E, et al. (2010); durante uma mesa de discussão entre especialistas americanos em saúde do homem foi explorado o porquê de os homens evitarem consultar-se com um médico. Essa não adesão relaciona-se a estereótipos de gênero baseados em características culturais que normatizam uma masculinidade hegemônica, onde a doença expressa à fragilidade do corpo e, por extensão, do seu portador. Apesar de alguns homens já se apresentarem mais abertos a serem examinados, ainda é necessário promover maior conscientização em relação à promoção de sua saúde.

De acordo com Al- haddad (2010); a temática do câncer foi apresentada em cinco trabalhos, onde dois tiveram o câncer de mama como foco. Para os homens, desenvolver uma doença culturalmente feminina é angustiante e estigmatizante, o que leva a não identificação de sinais precoces da doença. A maioria dos homens que são diagnosticados se encontra em estágios avançados e requerem tratamentos longos e com poucos resultados positivos.

Nesse contexto, a baixa prevalência da patologia nos homens resulta em uma atenção diminuída, inclusive dos profissionais de saúde. Estudo realizado com o objetivo de conhecer as informações apresentadas por homens com antecedentes familiares de câncer de mama, demonstrou o desconhecimento dos sintomas e sobre a realização do autoexame para identificação de nódulos na mama. Assim, existem barreiras a serem superadas, com destaque da importância de estratégias de prevenção para o controle dessa patologia, onde o autoexame da mama é apresentado como estratégia principal (THOMAS E, 2010).

No que se refere ao câncer de próstata, foi observada a preocupação dos homens com a exposição, o que dificulta o alcance dos objetivos dos programas de prevenção e promoção da saúde. Neste contexto, o toque retal ainda é uma medida pouco aceita, apesar do baixo custo, e estes indivíduos relatam que o exame desperta o medo da dor física e psicológica (OLIVEIRA P, et al., 2012).

De acordo com Gertrich C, et al. (2012); o estudo que investigou fatores dificultadores do rastreamento do câncer colorretal, desenvolvido nos Estados Unidos, o machismo foi destaque, com expressões de relutância dos homens, principalmente, em relação à colonoscopia. Este procedimento é considerado estigmatizante e potencialmente transformador. Ademais, os dados qualitativos apontaram a visão de que o exame poderia modificá-los de maneira fundamental, como representativo da perda de virgindade masculina.

Ao relacionar estilo de vida e saúde reprodutiva, é importante identificar fatores de risco modificáveis, como a circunferência abdominal, tabagismo ou consumo de álcool e atividade física. Estas características contribuem para o desenvolvimento de doenças crônicas, assim como para o declínio na saúde reprodutiva, sendo essencial o desenvolvimento de estratégias preventivas adequadas, em consonância com o desenvolvimento das políticas de saúde (HOLDEN C, et al., 2010).

Conforme Brasil, (2009); o PNAISH tem o propósito de qualificar a saúde da população masculina em relação aos cuidados que resguardem a integralidade da atenção, na qual é um dos princípios preconizado pelo SUS. Portanto, é necessário fortalecer e qualificar a atenção primária garantindo, assim, a promoção da saúde e a prevenção dos problemas evitáveis. Entretanto, o maior desafio é a resistência masculina, em ir à atenção primária, diante disso, aumenta não somente a sobrecarga financeira da sociedade, mas também, e sobretudo, o sofrimento físico e emocional do paciente e de sua família na luta pela conservação da saúde e por uma melhor qualidade de vida. 
Conforme Scussel MRR (2017); é notório que a PNAISH vem construindo um cenário para a discussão da saúde masculina e para a implementação de recursos de saúde que visam fortalecer a prevenção e promoção da saúde dos homens. Constitui-se este campo como um cenário rico em estudos sobre as particularidades masculinas, a percepção dos profissionais e os desafios a serem enfrentados neste contexto de atenção integral a saúde do homem. Entretanto, conclui-se que as ações de implementação da política ainda não foram suficientes para inserir efetivamente o público masculino nos serviços de saúde pela via de atenção primaria a saúde, chegando estes ainda hegemonicamente pelas portas dos serviços emergenciais e das especialidades, e com quadros de adoecimento já tornados crônicos ou em situações agudas.

Segundo Aguiar RS et al. (2015); é evidente a necessidade de capacitação e aprofundamento do conhecimento do conteúdo da PNAISH por parte dos enfermeiros, uma vez que o desconhecimento dessa política pode fazer com que eles sintam dificuldades em assistir à população masculina e atender as demandas a partir de suas características. É preciso permitir que os homens se sintam mais acolhidos para que percam o medo e desconstruam a ideia de invulnerabilidade que tem sobre si mesmo, para que dessa forma busquem pelos serviços de prevenção à saúde.

\section{Intervenções educativas direcionadas a população masculina}

As intervenções de saúde direcionadas aos homens utilizaram recursos diferenciados, como: palestras, stand com materiais educativos, oficinas, programa de tutoria e aconselhamento por telefone. A atenção ao horário em que as atividades foram ministradas revelou-se importante para a captação dos participantes, visto que um dos fatores apontados por estes para a não procura dos serviços de atenção primária é a sua posição de provedor.

Os homens alegam que o horário do funcionamento dos serviços de saúde coincide com a carga horária do trabalho. Visando romper essa barreira, foram desenvolvidas estratégias de educação em saúde no local de trabalho do público masculino. As ações obtiveram receptividade dos homens e constituíram atividades com representatividade e eficácia no alcance deste gênero (LEITE D, 2010; ILIC D, 2013).

Segundo Leite D. (2010); o estudo foi realizado com o objetivo de proporcionar a indivíduos do sexo masculino conhecimentos sobre aspectos globais da saúde desenvolveu as temáticas: qualidade de vida, problemas de coluna, depressão e estresse, diabetes, câncer de próstata e doenças cardiovasculares em um hospital e um centro automotivo brasileiros. Para tanto, foram ministradas palestras e entrega de folders. Ao término, ambas as intervenções foram avaliadas e mostraram impacto positivo no conhecimento sobre as doenças abordadas.

Conforme Ilic D (2013); na Austrália, foi desenvolvida a campanha Help a Mate em uma indústria de construção com a finalidade de conscientizar os homens sobre o câncer de próstata. Os conhecimentos sobre rastreamento e tratamento da patologia são limitados. O exame de sangue para identificação do antígeno específico da próstata e o exame do toque retal são reconhecidos como medidas primárias na detecção, porém, são poucas as informações que possuem sobre os mesmos.

Nessa perspectiva, foi criado nos Estados Unidos, em 2010, o Men's Health Forum. Anualmente, durante o evento são realizados gratuitamente consultas, exames e sessões de educação em saúde por profissionais médicos e educadores capacitados.

Esta é uma das razões apontadas como atrativas, visto que para os homens é oportunidade de atendimento a um número maior de problemas em um único momento. Além disso, voluntários bilíngues ampliam o acesso a indivíduos de diferentes origens e culturas (GRANT C, et al., 2012).

De acordo com Fontes L et al. (2011); no âmbito da Atenção Primária de Saúde, foram desenvolvidas estratégias de educação em saúde para homens da Paraíba, Brasil. A intervenção consistiu na construção de stand com materiais educativos sobre prevenção de agravos e promoção da saúde, aliados aos serviços de aferição da pressão arterial e medida da circunferência abdominal dos participantes do evento. A iniciativa do centro universitário fortaleceu a interlocução entre ensino, serviços de saúde e os princípios da Política Nacional Integral de saúde do Homem. 
Conforme Foster L (2012); além das modalidades de educação em saúde descritas, estudo desenvolvido na Nova Zelândia proporcionou um evento noturno com o objetivo de promover a saúde de homens maiores de 35 anos e que não frequentavam os serviços de saúde. A ação teve como foco a conscientização do risco cardiovascular, encorajamento à proatividade e enfrentamento de problemas de próstata, depressão e violência familiar, reconhecidos como de difícil tratamento, assim como a apreciação da paternidade. Foram realizadas avaliações individuais de saúde por enfermeiras e encaminhamentos a centros de saúde.

Segundo Constanza M, et al. (2011); outro tipo de intervenção identificada foi o Computer-Assisted Telephone Counseling (CATC) com objetivo de incentivar a decisão dos homens em realizar o exame do antígeno específico da próstata. Os indivíduos receberam folheto informativo contendo conceitos que urologistas e oncologistas consideram chave para entender o câncer de próstata e o teste, posterior a adequações realizadas por um revisor de menor escolaridade. O CATC forneceu suporte de decisão para os homens com cientificidade e efetividade, sendo recurso com potencial para discussão e utilização em maior escala.

Estudo realizado na Austrália avaliou a eficácia de programas de tutoria desenvolvidos pelos Men's Sheds e os fatores que refletem um ambiente inclusivo e com foco na saúde. Os mentores dessas tutorias eram os membros mais antigos dos Galpões de Homens ou um instrutor qualificado e as temáticas abordadas foram: diabetes, atividade física, alimentação saudável e primeiros socorros (CORDIER R e WILSON N, 2014). As estratégias de educação em saúde do homem por meio de intervenções produziram um impacto positivo na vida dos homens, com resultados relevantes sobre o conhecimento das patologias que assolam.

\section{Ações direcionadas ao contexto profissional}

Foram poucos os trabalhos, neste estudo, que abordaram intervenções educativas no âmbito profissional. Um artigo trabalhou a formação de estudantes com vistas à melhoria do desempenho em atividades assistenciais e outro teve foco na capacitação de profissionais de saúde.

Conforme Fairbanck C (2011); foi desenvolvido um estudo na Austrália com o objetivo de ensinar aos 30 alunos do curso de medicina de uma universidade, durante sua rotação urológica, habilidades de comunicação e técnicas de exames genitais, retais e de hérnia, utilizando vídeos educativos. $O$ instrumento consistiu em um tutorial elaborado por meio do recrutamento de homens da comunidade e abordou a fundamentação teórica e procedimental, além de treinarem estas habilidades de feedback. Os estudantes foram avaliados antes e depois da exibição e os resultados demonstraram o aumento do nível de conhecimento e de confiança na atuação destas problemáticas de saúde do homem.

Nesse sentido, de acordo com Mccullagh J (2011); foi desenvolvido um programa nacional na Inglaterra para treinamento em saúde do homem para profissionais de saúde e assistência social para lidar com as desigualdades de gênero e mediar a prestação de cuidados efetiva. O estudo consistiu no curso de formação de 38 profissionais com duração de um dia, no qual foram trabalhadas as temáticas de desigualdade na saúde dos homens, questões de gênero, perspectivas de saúde dos homens, efeitos do trabalho na saúde, prevenção de riscos e fatores que influenciam a utilização dos serviços pelos homens.

Após o treinamento, segundo Mccullagh J (2011); foi aplicado um questionário para todos os participantes a fim de se avaliar a eficácia do curso, os níveis de satisfação e impacto dessa medida na prática futura de trabalho. A avaliação dos resultados demonstrou que o treinamento foi bem recebido pelos participantes que afirmaram o cumprimento do curso aos requisitos de conteúdo e didática. Todos os participantes intencionaram adotar em suas práticas de trabalho uma perspectiva voltada para o gênero masculino e afirmaram que recomendariam o curso para outros colegas de saúde pública.

Conforme Silveira CLG, et al. (2017); observa-se que mesmo após a implantação da Política Nacional de saúde do homem, não existem ações contínuas no serviço de saúde, na atenção primária voltadas para o gênero masculino, pois a falta de conhecimento e de treinamento sobre a política de atenção à saúde do homem e a falta de estrutura do serviço, como citada pelos profissionais, contribuem para que os homens não procurem a unidade de saúde, além dos obstáculos levantados pelos próprios homens. 
A promoção da saúde precisa, também, do apoio de outros setores da sociedade - como as escolas, que, por serem fontes de aprendizado e formadoras de opinião, podem colaborar com a educação sobre saúde. Assim, pode-se fomentar o entendimento sobre a importância desse assunto, buscando uma saúde coletiva de excelência e menos dispendiosa à nação (TRILICO MLC, 2015).

\section{CONCLUSÃO}

A pesquisa possibilitou, através da revisão integrativa da literatura, reconhecer as principais temáticas relacionados à saúde do homem incluindo patologias, tais como câncer de próstata, distúrbios reprodutivos. Portanto, é necessário que os profissionais tenham os devidos conhecimentos técnicos científicos para o desenvolvimento de ações de educação em saúde, com objetivo de instruir esse gênero sobre a sua própria saúde levando-os buscar assistência em saúde na Atenção Básica e o rompimento das diversas barreiras que os afastam desse serviço. Para tanto, a intervenções de educação em saúde devem ser multifacetadas: sair em busca do público masculino nos locais de trabalho ou na comunidade; serem realizadas fora do horário comercial e por vezes adaptarem o espaço físico da intervenção na tentativa de atrair a atenção desses homens. Contudo, a produção científica evidenciou que há lacunas existentes no conhecimento produzido sobre a temática abordada. E que se faz necessário futuros estudos nesse tema, a fim de aprofundar o conhecimento científico.

\section{REFERÊNCIAS}

1. AGUIAR RS, et al, A percepção do enfermeiro da estratégia saúde da família sobre a saúde do homem. R. Enferm. Cent. O. Min. v.5, n.3, p1844-54, 2015.

2. AL HM. Breast cancer in men: the importance of teaching and raising awareness. Clin J Oncol Nurs. v.14, n.1, p.:31-2,2010.

3. BERTOLINI DNP, SIMONETTI JP. O Gênero Masculino e os Cuidados de Saúde: a Experiência de Homens de um Centro de Saúde. Rev. Esc. Ana Nery, Rio de Janeiro, v. 18, n.4, p. 722-727, 2014.

4. BRASIL. Ministério da Saúde. Política Nacional de Atenção Integral à Saúde do Homem: princípios e diretrizes. Brasília: MS, 2009.

5. CORDIER R, WILSON N. Mentoring at Men's Sheds: an international survey about a community approach to health and well-being. Health Soc Care Community. v.22, n.3, p.249-58, 2014.

6. COSTANZA M, et al. Helping men make an informed decision about prostate cancer screening: a pilot study of telephone counseling. Patient Educ Couns. v.82, n.2, p.193-200, 2011.

7. FAIRBANCK C. Men's health: it is imperative to teach scrotal and rectal examination. Clin Teach. v.8, n.2, p.101-4, 2011.

8. FONTES W, et al. Atenção à saúde do homem: interlocução entre ensino e serviço. Acta paul enferm. v.24, n.3, p.430-433, 2011.

9. FOSTER L. Raising mens' awareness of the need to monitor their health. Nurs N Z v.18, n.3, p.16-7, 2012.

10. GETRICH C, et al. Expressions of machismo in colorectal cancer screening among New Mexico Hispanic subpopulations. Qual Health Res. v.22, n.4, p.546-59, 2012.

11. GRANT C, et al. The men's health forum: an initiative to address health disparities in the community. J Community Health. v.37, n.4, p773-80. 2012.

12. HOLDEN C, et al. Determinants of male reproductive health disorders: The Men in Australia Telephone Survey (MATeS). BMC Public Health. v.24, n.10, p.96, 2010

13. ILIC D. Educating men about prostate cancer in the workplace. Am J Mens Health v.7, n.4, p.285-94, 2013.

14. LEITE D, et al. A influência de um programa de educação na saúde do homem. Mundo saúde. v.34. n.1, p.50-56, 2013.

15. MACHIN R et al. Concepções de gênero, masculinidade e cuidados em saúde: estudo com profissionais de saúde da atenção primária. Ciência \& Saúde Coletiva. v.16, n.11, p. 4503-4512, 2011.

16. MCCULLAGH J. The invisible man - Development of a national men's health training programme for public health practitioners: challenges and successes. Public Health. v.125, p.7, n.401-6, 2011.

17. MENDES K, et al. Revisão integrativa: método de pesquisa para a incorporação de evidências na saúde e na enfermagem. Texto Contexto Enferm v.17, p.4, n.758-764, 2008.

18. MOURA EC, et al. Percepções Sobre a Saúde dos Homens Numa Perspectiva Relacional de Gênero, Brasil, 2014. Rev. Ciências e Saúde Coletiva, v. 22, n. 1, p 291-300, 2017.

19. OLIVEIRA $P$, et al. The workers knowledge about prostate cancer: a descriptive exploratory study. Online Braz J Nurs. v.11, n.1, 2012.

20. POZZATI R, et al. O cuidado na saúde dos homens: realidade e perspectivas. Rev enferm UERJ. v.21, n.4, p.5405, 2013. 
21. SCUSSEL MRR, MACHADO DM. Política nacional de assistência integral à saúde do homem: uma revisão integrativa. REFACS (online). v.5, n.2, p.235-244, 2017.

22. SILVEIRA CLG, et al. Atenção à saúde do homem na atenção primária em saúde: uma revisão integrativa. Rev enferm UFPE on line., v.11, n.Supl. 3, p.1528-9, 2017.

23. STORINO LP, et al. Necessidades de saúde de homens na atenção básica: acolhimento e vínculo como potencializadores da integralidade. Esc. Anna Nery, v. 17, n. 4, p. 638-645, 2013.

24. THOMAS E. Men's awareness and knowledge of male breast cancer. Am J Nurs. v.110, n.10, p.32-7, 2010.

25. TRILICO MLC, et al. Discursos masculinos sobre prevenção e promoção da saúde do homem. Trab. educ. saúde. v.13, n.2, 2015

26. VIEIRA K, et al. Atendimento da população masculina em unidade básica saúde da família: motivos para a (não) procura. Esc Anna Nery v.17, n.1, p.120-127, 2013.

27. WHO. World Health Organization. Milestones in Health Promotion Statements from Global Conferences. Gevena: World Health Organization, 2009

28. YARNELL E, et al. Roundtable discussion: focus on men's health. Altern Complement Ther. v.16, n.2, p.77-82, 2010. 